\title{
Resveratrol: an effective pharmacological agent to prevent inflammation-induced atrial fibrillation?
}

\author{
Henry Sutanto ${ }^{1} \cdot$ Dobromir Dobrev $^{2} \cdot$ Jordi Heijman $^{1}$ (D) \\ Received: 7 September 2018 / Accepted: 11 September 2018 / Published online: 20 September 2018 \\ (C) Springer-Verlag GmbH Germany, part of Springer Nature 2018
}

Atrial fibrillation (AF) affects $>33.5$ million people worldwide, a number that is expected to increase significantly in the upcoming years due to the aging of the population and the increased prevalence of risk factors such as hypertension and diabetes (Andrade et al. 2014; Chugh et al. 2014). AF has important clinical consequences, negatively affecting quality of life and doubling cardiovascular morbidity and mortality (Kirchhof et al. 2016). Currently available pharmacological anti-AF therapies are only moderately effective, and their use is limited by severe adverse effects, including ventricular proarrhythmia and extracardiac toxicity (Calvo et al. 2018; Dan and Dobrev 2018; Lafuente-Lafuente et al. 2015). The limited efficacy of these pharmacological agents is likely due to a one-size-fits-most approach despite the diverse etiology of $\mathrm{AF}$, which can occur in a wide range of conditions as the result of multiple pathologic processes, including $\mathrm{Ca}^{2+}$-handling abnormalities, electrical remodeling, structural remodeling, and autonomic imbalance (Heijman et al. 2016; Heijman et al. 2018; Nattel and Harada 2014). In addition, the high prevalence of asymptomatic AF hinders AF detection and initiation of anti-AF therapies early during the disease process, when AF is still amenable to therapy (Boriani et al. 2015; Siontis et al. 2016).

AF can also be promoted by increased inflammatory signaling and systemic inflammatory disease (Lazzerini et al. 2014; Lindhardsen et al. 2012; Yao et al. 2018), including rheumatoid arthritis (RA). In a Danish nationwide cohort

Editorial to: Zhang et al. Resveratrol prevents atrial fibrillation by inhibiting atrial structural and metabolic remodeling in collageninduced arthritis rats.

Jordi Heijman

jordi.heijman@maastrichtuniversity.nl

1 Department of Cardiology, CARIM School for cardiovascular diseases, Maastricht University, PO Box 616, 6200 MD Maastricht, The Netherlands

2 Institute of Pharmacology, West German Heart and Vascular Center, University Duisburg-Essen, Essen, Germany study (Lindhardsen et al. 2012), the incidence of AF was $40 \%$ higher in RA patients compared to the general population, with higher relative risks in younger patients. Similarly, Kim et al. (2014) reported an AF incidence-rate ratio of 1.4 in RA patients, although this association disappeared after correcting for a wide range of other cardiovascular risk factors. Thus, the higher propensity of AF in RA may be attributed to several pathophysiological mechanisms, involving both direct and indirect effects of systemic inflammation on the atria. The indirect AF-promoting effects include the development of heart failure and ischemic heart disease, which subsequently initiate atrial structural, autonomic, and electromechanical remodeling (Dai et al. 2017; Lazzerini et al. 2014; Li and Dobrev 2018; Nattel and Harada 2014). Furthermore, increased arterial stiffness and drugs used for the treatment of RA may also modulate the interaction between RA and AF (Lindhardsen et al. 2012). Thus, acute and chronic inflammation can directly promote AF through several pathways. Studies on the mechanisms of postoperative AF have shown that acute inflammation can induce AF by promoting cellular oxidative stress via pro-inflammatory cytokines (see Fig. 1) (Lubbers et al. 2015; Maesen et al. 2012). Conversely, chronic inflammation promotes AF through atrial structural remodeling, particularly the development of atrial fibrosis (Abe et al. 2018; Chen et al. 2018).

In the current issue of Naunyn-Schmiedeberg's Archives of Pharmacology, Zhang and colleagues (Zhang et al. 2018) investigated the effect of resveratrol, a bioactive polyphenol commonly found in wine, grapes, mulberries, and peanuts (Baczko and Light 2015), on atrial structural and metabolic remodeling, as well as AF-susceptibility, in rats with collagen-induced arthritis (CIA). AF inducibility and duration were markedly elevated in CIA rats and were accompanied by increased atrial levels of IL- 6 and TNF- $\alpha$, as well as atrial apoptosis and fibrosis. CIA also downregulated the AMPK/PGC-1 $\alpha$ pathway, suggesting dysregulation of atrial metabolism. Zhang et al. (2018) also showed that 4 weeks of resveratrol treatment started 3 weeks after the second collagen injection could attenuate 


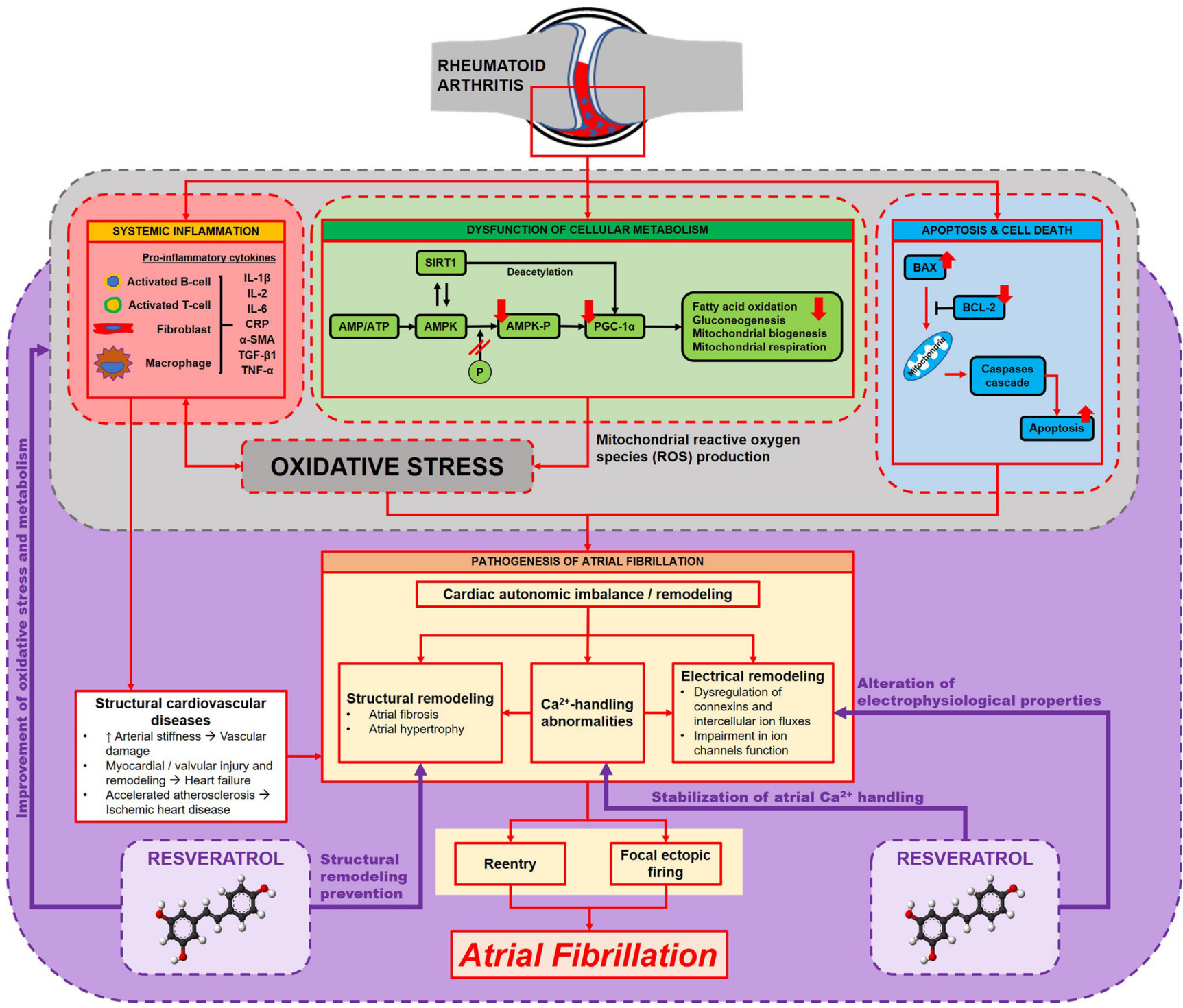

Fig. 1 Schematic illustration of the pathophysiology of rheumatoid arthritis (RA)-induced atrial fibrillation (AF) and potential antiarrhythmic effects of resveratrol. Resveratrol exerts its capability to prevent RA-induced AF through several pathophysiological processes, including inflammation, altered metabolism, oxidative stress, and $\mathrm{Ca}^{2+}$

all proarrhythmic components of the CIA-induced substrate and significantly reduced the incidence and duration of burst-pacing-induced AF.

Resveratrol is a natural polyphenol derivative that has been widely investigated and is believed to have cardioprotective and antiarrhythmic properties (Baczko and Light 2015; Baczko et al. 2014; Frommeyer et al. 2018; Liu et al. 2017; Trevino-Saldana and Garcia-Rivas 2017). Consistent with the findings by Zhang et al. (2018), several studies have shown that resveratrol is also a promising pharmacological agent for the treatment of AF (Baczko and Light 2015). However, the potential mechanisms underlying the anti-AF effects of resveratrol are diverse (Fig. 1), and their relative contribution in different forms of AF is unknown. handling/signaling in cardiomyocytes, as well as by altering several electrophysiological properties, thereby targeting structural and electrical remodeling. Red lines represent the pathophysiology of RA-induced AF, and purple lines represent potential targets of resveratrol

Resveratrol can alter cardiac electrophysiology and arrhythmogenesis through a direct effect on cardiac ion channels, including inhibition of the peak and late components of cardiac voltage-gated $\mathrm{Na}^{+}$channels (Baczko and Light 2015; Frommeyer et al. 2018). Resveratrol increases myofilament $\mathrm{Ca}^{2+}$ sensitivity but inhibits L-type $\mathrm{Ca}^{2+}$ currents, resulting in a decreased $\mathrm{Ca}^{2+}$ transient amplitude (Liu et al. 2017). It also inhibits phosphodiesterases, enhancing the inotropic effect of sympathomimetic agents, while inhibiting their proarrhythmic effects (Hernandez-Cascales 2017). Acute resveratrol treatment reduces the number of inducible AF episodes in an ex vivo rabbit AF model, without significant reduction in AF susceptibility (Frommeyer et al. 2018). In this model, resveratrol induces slowing of intrinsic heart rate, 
slowing of conduction velocity, and prolongation of atrial effective refractory period (aERP). Similarly, a resveratrol derivative with increased affinity for Kv1.5, underlying the atrial-specific ultrarapid delayed-rectifier $\mathrm{K}^{+}$current, has been shown to prolong aERP and reduce the duration of AF episodes in a dog model of atrial tachycardia remodeling (Baczko et al. 2014). This resveratrol-derived small molecule did not prolong ventricular repolarization, suggesting a low risk for Torsade de Pointes as a complication of AF treatment (Baczko et al. 2014). AF inducibility is also increased in a rabbit model of coronary-ligation-induced heart failure, but in this model, atrial ERP is prolonged and both AF inducibility and ERP are decreased by acute application of resveratrol. Similarly, acute resveratrol application reduces ventricular ERP in guinea pig ventricular cardiomyocytes (Liew et al. 2005). In contrast, in the study by Zhang et al. (2018), long-term treatment with resveratrol did not significantly affect aERP in CIA rats. The latter may be due to species-, chamber-, or disease-specific effects, as well as compensatory electrical remodeling during long-term treatment, which is absent during acute resveratrol application.

Indeed, long-term resveratrol treatment can have antiarrhythmic effects by preventing atrial remodeling. For example, 1 week of daily resveratrol treatment, initiated 4 weeks after coronary ligation in the rabbit heart failure module attenuates atrial apoptosis and fibrosis and, partially, normalizes mRNA levels of several ion channels, as well as protein levels of SERCA2a and phospholamban, through a PI3K/AKT/ eNOS pathway (Chong et al. 2015). Consistent with this normalization of atrial electrical, $\mathrm{Ca}^{2+}$-handling and structural remodeling, long-term resveratrol treatment also reduced $\mathrm{AF}$ inducibility. Resveratrol also attenuates hypertrophic remodeling and can prevent the development of heart failure through modulation of $\mathrm{Ca}^{2+}$-signaling pathways, notably by inhibiting calcineurin and $\mathrm{Ca}^{2+} /$ calmodulin-dependent protein kinase-II (CaMKII) activation (Baczko and Light 2015; Liu et al. 2017), which may both directly and indirectly affect atrial remodeling. For example, in rats with aortic banding, longterm resveratrol treatment prevents $\mathrm{Ca}^{2+}$-handling remodeling, including increased CaMKII expression and autophosphorylation, as well as fibrosis and ventricular dilation (Dong et al. 2014; Liu et al. 2017). Finally, resveratrol is a potent anti-oxidant, reducing cellular oxidative stress, which represents a major trigger for electrical and $\mathrm{Ca}^{2+}$-handling abnormalities, including hyperactive ryanodine receptormediated sarcoplasmic reticulum $\mathrm{Ca}^{2+}$ releases that may initiate AF (Liu et al. 2017), as well as for metabolic and structural remodeling (Harada et al. 2017; Harada et al. 2015). In agreement, in the study by Zhang et al. (2018), resveratrol restores the CIA-induced decrease in phosphorylation and expression of AMPK, normalizing atrial metabolism, including the expression of PGC- $1 \alpha$, an important regulator of mitochondria bioenergetics, and the main energy transport proteins Glut4 and FAT/CD36 (Zhang et al. 2018). These alterations, together with the reduction in atrial inflammation, likely contribute to the resveratrol-induced decrease in cellular apoptosis and fibrosis observed in this study. Taken together, the work by Zhang et al. (2018) suggests that resveratrol can prevent the development of AF-maintaining structural remodeling through beneficial modulation of atrial inflammation and metabolism.

Despite the potential of resveratrol as antiarrhythmic agent, several questions need to be addressed. Firstly, although Zhang et al. (2018) show that resveratrol can suppress the progression of the AF-promoting substrate that develops during the final 4 weeks of drug administration initiated 3 weeks after the last collagen injection, the magnitude of atrial remodeling generated before the administration of resveratrol is unknown. Typically, notable atrial structural remodeling only develops after 8-14 weeks of interventions (Dai et al. 2017; Kim et al. 2011). Thus, it is unclear whether resveratrol can "only" prevent the development of atrial remodeling or can actually revert existing atrial structural remodeling, which would be more clinically relevant, since resveratrol will likely be prescribed to patients who already have a significant degree of atrial remodeling. Secondly, resveratrol has a limited halflife ( $\pm 49 \mathrm{~min}$ in normal individuals (Amiot et al. 2013; Baczko and Light 2015)), as it is rapidly metabolized and cleared from the body, making it unsuitable for oral administration. Although this half-life can be improved (Amiot et al. 2013), achieving a stable pharmacokinetic profile may still be challenging due to accompanying systemic diseases that commonly develop together with RA-induced AF, including impaired renal function and other autoimmune disorders (Kochi et al. 2018). Thirdly, although resveratrol is generally considered a safe drug, its electrophysiological effects may still present a potential arrhythmogenic risk, particularly at high concentrations (Liu et al. 2017; Trevino-Saldana and GarciaRivas 2017). For example, polydatin, a drug highly similar to resveratrol, increases ryanodine receptor activity, leading to more $\mathrm{Ca}^{2+}$ sparks and proarrhythmic spontaneous $\mathrm{Ca}^{2+}$ releases from the sarcoplasmic reticulum (Liu et al. 2017). Finally, most of the antiarrhythmic effects of resveratrol have only been documented in animal models. Although some indirect studies have hinted at a beneficial effect of resveratrol in patients, this has been questioned by recent randomized controlled trials (Kjaer et al. 2017). Whether the same antiarrhythmic effects observed with resveratrol in animal models can also be achieved in AF patients (either with or without RA) is unknown, which highlights the importance of future investigations of such effects in patient samples and clinical studies.

Taken together, resveratrol represents a potentially novel antiarrhythmic agent with an apparently strong safety profile. Many publications have shown that resveratrol targets, either directly or indirectly, multiple substrates involved in the initiation and maintenance of $\mathrm{AF}$, including $\mathrm{Ca}^{2+}$-handling 
abnormalities and atrial electrical and structural remodeling. Thus, resveratrol may offer new therapeutic approaches for different forms of $\mathrm{AF}$, including $\mathrm{AF}$ in the setting of RA. However, despite the potential of resveratrol, extensive further experimental research and clinical trials are needed to confirm these antiarrhythmic effects in patients with $\mathrm{AF}$.

Author contribution HS and JH drafted the editorial. DD critically revised the editorial. All authors approved the final version.

Funding information The authors are supported by the Netherlands Organization for Scientific Research (ZonMW Veni 91616057 to JH), the National Institutes of Health (R01-HL131517 and R01-HL136389 to DD), and the German Research Foundation (DFG, Do 769/4-1 to DD).

\section{Compliance with ethical standards}

Human and animal studies This editorial does not contain any new human or animal data.

Conflicts of interest The authors declare that they have no conflicts of interest.

\section{References}

Abe I, Teshima Y, Kondo H, Kaku H, Kira S, Ikebe Y, Saito S, Fukui A, Shinohara T, Yufu K, Nakagawa M, Hijiya N, Moriyama M, Shimada T, Miyamoto S, Takahashi N (2018) Association of fibrotic remodeling and cytokines/chemokines content in epicardial adipose tissue with atrial myocardial fibrosis in patients with atrial fibrillation. Heart Rhythm. https://doi.org/10.1016/j.hrthm.2018.06.025

Amiot MJ, Romier B, Anh Dao TM, Fanciullino R, Ciccolini J, Burcelin R, Pechere L, Emond C, Savouret JF, Seree E (2013) Optimization of trans-resveratrol bioavailability for human therapy. Biochimie 95: 1233-1238. https://doi.org/10.1016/j.biochi.2013.01.008

Andrade J, Khairy P, Dobrev D, Nattel S (2014) The clinical profile and pathophysiology of atrial fibrillation: relationships among clinical features, epidemiology, and mechanisms. Circ Res 114:14531468. https://doi.org/10.1161/CIRCRESAHA.114.303211

Baczko I, Light PE (2015) Resveratrol and derivatives for the treatment of atrial fibrillation. Ann N Y Acad Sci 1348:68-74. https://doi.org/10. 1111/nyas. 12843

Baczko I, Liknes D, Yang W, Hamming KC, Searle G, Jaeger K, Husti Z, Juhasz V, Klausz G, Pap R, Saghy L, Varro A, Dolinsky V, Wang S, Rauniyar V, Hall D, Dyck JRB, Light PE (2014) Characterization of a novel multifunctional resveratrol derivative for the treatment of atrial fibrillation. Br J Pharmacol 171:92-106. https://doi.org/10. 1111/bph.12409

Boriani G, Laroche C, Diemberger I, Fantecchi E, Popescu MI, Rasmussen LH, Sinagra G, Petrescu L, Tavazzi L, Maggioni AP, Lip GYH (2015) Asymptomatic atrial fibrillation: clinical correlates, management, and outcomes in the EORP-AF Pilot General Registry. Am J Med 128 e502:509-518. https://doi.org/10.1016/j.amjmed.2014.11.026

Calvo D, Filgueiras-Rama D, Jalife J (2018) Mechanisms and drug development in atrial fibrillation. Pharmacol Rev 70:505-525. https:// doi.org/10.1124/pr.117.014183

Chen G, Chelu MG, Dobrev D, Li N (2018) Cardiomyocyte Inflammasome signaling in cardiomyopathies and atrial fibrillation: mechanisms and potential therapeutic implications. Front Physiol 9: 1115. https://doi.org/10.3389/fphys.2018.01115
Chong E, Chang SL, Hsiao YW, Singhal R, Liu SH, Leha T, Lin WY, Hsu CP, Chen YC, Chen YJ, Wu TJ, Higa S, Chen SA (2015) Resveratrol, a red wine antioxidant, reduces atrial fibrillation susceptibility in the failing heart by $\mathrm{PI} 3 \mathrm{~K} / \mathrm{AKT} / \mathrm{eNOS}$ signaling pathway activation. Heart Rhythm 12:1046-1056. https://doi.org/10. 1016/j.hrthm.2015.01.044

Chugh SS, Havmoeller R, Narayanan K, Singh D, Rienstra M, Benjamin EJ, Gillum RF, Kim YH, McAnulty JH, Zheng ZJ, Forouzanfar MH, Naghavi M, Mensah GA, Ezzati M, Murray CJL (2014) Worldwide epidemiology of atrial fibrillation: a Global Burden of Disease 2010 Study. Circulation 129:837-847. https://doi.org/10.1161/ CIRCULATIONAHA.113.005119

Dai H, Wang X, Yin S, Zhang Y, Han Y, Yang N, Xu J, Sun L, Yuan Y, Sheng L, Gong Y, Li Y (2017) Atrial fibrillation promotion in a rat model of rheumatoid arthritis. J Am Heart Assoc 6:e007320. https:// doi.org/10.1161/JAHA.117.007320

Dan GA, Dobrev D (2018) Antiarrhythmic drugs for atrial fibrillation: imminent impulses are emerging. Int J Cardiol Heart Vasc. https:// doi.org/10.1016/j.ijcha.2018.08.005

Dong Q, Wu Z, Li X, Yan J, Zhao L, Yang C, Lu J, Deng J, Chen M (2014) Resveratrol ameliorates cardiac dysfunction induced by pressure overload in rats via structural protection and modulation of $\mathrm{Ca}^{2+}$ cycling proteins. J Transl Med 12:323. https://doi.org/10. 1186/s12967-014-0323-x

Frommeyer G, Wolfes J, Ellermann C, Kochhauser S, Dechering DG, Eckardt L (2018) Acute electrophysiologic effects of the polyphenols resveratrol and piceatannol in rabbit atria. Clin Exp Pharmacol Physiol. https://doi.org/10.1111/1440-1681.13005

Harada M, Melka J, Sobue Y, Nattel S (2017) Metabolic considerations in atrial fibrillation-mechanistic insights and therapeutic opportunities. Circ J 81:1749-1757. https://doi.org/10.1253/circj.CJ-17-1058

Harada M, Van Wagoner DR, Nattel S (2015) Role of inflammation in atrial fibrillation pathophysiology and management. Circ J 79:495502. https://doi.org/10.1253/circj.CJ-15-0138

Heijman J, Algalarrondo V, Voigt N, Melka J, Wehrens XH, Dobrev D, Nattel S (2016) The value of basic research insights into atrial fibrillation mechanisms as a guide to therapeutic innovation: a critical analysis. Cardiovasc Res 109:467-479. https://doi.org/10.1093/cvr/ cvv275

Heijman J, Guichard JB, Dobrev D, Nattel S (2018) Translational challenges in atrial fibrillation. Circ Res 122:752-773. https://doi.org/ 10.1161/CIRCRESAHA.117.311081

Hernandez-Cascales J (2017) Resveratrol enhances the inotropic effect but inhibits the proarrhythmic effect of sympathomimetic agents in rat myocardium. PeerJ 5:e3113. https://doi.org/10.7717/peerj.3113

Kim SC, Liu J, Solomon DH (2014) The risk of atrial fibrillation in patients with rheumatoid arthritis. Ann Rheum Dis 73:1091-1095. https://doi.org/10.1136/annrheumdis-2013-203343

Kim SJ, Choisy SC, Barman P, Zhang H, Hancox JC, Jones SA, James AF (2011) Atrial remodeling and the substrate for atrial fibrillation in rat hearts with elevated afterload. Circ Arrhythm Electrophysiol 4: 761-769. https://doi.org/10.1161/CIRCEP.111.964783

Kirchhof P, Benussi S, Kotecha D, Ahlsson A, Atar D, Casadei B, Castella M, Diener HC, Heidbuchel H, Hendriks J, Hindricks G, Manolis AS, Oldgren J, Popescu BA, Schotten U, van Putte B, Vardas P, Agewall S, Camm J, Baron Esquivias G, Budts W, Carerj S, Casselman F, Coca A, de Caterina R, Deftereos S, Dobrev D, Ferro JM, Filippatos G, Fitzsimons D, Gorenek B, Guenoun M, Hohnloser SH, Kolh P, Lip GYH, Manolis A, McMurray J, Ponikowski P, Rosenhek R, Ruschitzka F, Savelieva I, Sharma S, Suwalski P, Tamargo JL, Taylor CJ, van Gelder IC, Voors AA, Windecker S, Zamorano JL, Zeppenfeld K (2016) 2016 ESC guidelines for the management of atrial fibrillation developed in collaboration with EACTS. Eur Heart J 37:2893-2962. https:// doi.org/10.1093/eurheartj/ehw210 
Kjaer TN et al (2017) No beneficial effects of resveratrol on the metabolic syndrome: a randomized placebo-controlled clinical trial. J Clin Endocrinol Metab 102:1642-1651. https://doi.org/10.1210/jc. 2016-2160

Kochi M, Kohagura K, Shiohira Y, Iseki K, Ohya Y (2018) Chronic kidney disease, inflammation, and cardiovascular disease risk in rheumatoid arthritis. J Cardiol 71:277-283. https://doi.org/10. 1016/j.jjcc.2017.08.008

Lafuente-Lafuente C, Valembois L, Bergmann JF, Belmin J (2015) Antiarrhythmics for maintaining sinus rhythm after cardioversion of atrial fibrillation Cochrane Database. Syst Rev:CD005049. https://doi.org/10.1002/14651858.CD005049.pub4

Lazzerini PE, Capecchi PL, Acampa M, Galeazzi M, Laghi-Pasini F (2014) Arrhythmic risk in rheumatoid arthritis: the driving role of systemic inflammation. Autoimmun Rev 13:936-944. https://doi. org/10.1016/j.autrev.2014.05.007

Li N, Dobrev D (2018) Targeting atrial fibrillation promoting atrial structural remodeling: is this a viable strategy in patients with heart failure? Naunyn Schmiedeberg's Arch Pharmacol 391:231-233. https:// doi.org/10.1007/s00210-018-1463-y

Liew R, Stagg MA, MacLeod KT, Collins P (2005) The red wine polyphenol, resveratrol, exerts acute direct actions on Guinea-pig ventricular myocytes. Eur J Pharmacol 519:1-8. https://doi.org/10. 1016/j.ejphar.2005.06.017

Lindhardsen J, Ahlehoff O, Gislason GH, Madsen OR, Olesen JB, Svendsen JH, Torp-Pedersen C, Hansen PR (2012) Risk of atrial fibrillation and stroke in rheumatoid arthritis: Danish nationwide cohort study. BMJ 344:e1257. https://doi.org/10. 1136/bmj.e1257
Liu W, Chen P, Deng J, Lv J, Liu J (2017) Resveratrol and polydatin as modulators of $\mathrm{Ca}^{2+}$ mobilization in the cardiovascular system. Ann N Y Acad Sci 1403:82-91. https://doi.org/10.1111/nyas. 13386

Lubbers ER, Murphy NP, Mohler PJ (2015) Defining the links between oxidative stress-based biomarkers and postoperative atrial fibrillation. J Am Heart Assoc 4:e002110. https://doi.org/10.1161/JAHA. 115.002110

Maesen B, Nijs J, Maessen J, Allessie M, Schotten U (2012) Postoperative atrial fibrillation: a maze of mechanisms. Europace 14: 159-174. https://doi.org/10.1093/europace/eur208

Nattel S, Harada M (2014) Atrial remodeling and atrial fibrillation: recent advances and translational perspectives. J Am Coll Cardiol 63: 2335-2345. https://doi.org/10.1016/j.jacc.2014.02.555

Siontis KC, Gersh BJ, Killian JM, Noseworthy PA, McCabe P, Weston SA, Roger VL, Chamberlain AM (2016) Typical, atypical, and asymptomatic presentations of new-onset atrial fibrillation in the community: characteristics and prognostic implications. Heart Rhythm 13:1418 1424. https://doi.org/10.1016/j.hrthm.2016.03.003

Trevino-Saldana N, Garcia-Rivas G (2017) Regulation of sirtuinmediated protein deacetylation by cardioprotective phytochemicals. Oxidative Med Cell Longev 2017:1750306-1750316. https://oi. org/10.1155/2017/1750306

Yao C et al (2018) Enhanced cardiomyocyte NLRP3 inflammasome signaling promotes atrial fibrillation. Circulation. https://doi.org/10. 1161/CIRCULATIONAHA.118.035202

Zhang Y, Zhang S, Liu Z, Zhao X, Yuan Y, Sheng L, Li Y (2018) Resveratrol prevents atrial fibrillation by inhibiting atrial structural and metabolic remodeling in collagen-induced arthritis rats. Naunyn Schmiedeberg's Arch Pharmacol doi:https://doi.org/10.1007/ s00210-018-1554-9 